\title{
$\vee 94-24455$
}

1993

NASA/ASEE SUMMER FACULTY FELLOWSHIP PROGRAM

MARSHALL SPACE FLIGHT CENTER

THE UNIVERSITY OF ALABAMA IN HUNTSVILLE

USING CONTOUR MAPS TO SEARCH FOR

RED-SHIFTED $511 \mathrm{keV}$ FEATURES

IN BATSE GRB SPECTRA

Prepared By:

Academic Rank:

Institution and

Department:

MSFC Colleague:

NASA/MSFC:

Laboratory:

Division:

Branch:
Peter G. Varmette

Graduate Student

Mississippi State University

Department of Physics

and Astronomy

Gerald Fishman, Ph.D.

Space Science

Astrophysics

Gamma-Ray Astronomy

L 
Since their discovery twenty years ago, the origin of gamma-ray bursts (GRB's) has remained an intriguing mystery. The quest to understand these objects has given rise to a plethora of competing theories. Several theories suggest that GRB's are galactic in origin while others suggest that GRB's are cosmological (Harding 1993).

One piece of evidence that might provide scientists with a key to understanding the origin of GRB's may be whether or not spectral emission and absorption features exist in burst spectra. If the features exist and can be attributed to either cyclotron lines or to red-shifted $511 \mathrm{keV}$ annihilation lines then credence would be given to those theories that support a galactic origin, i.e. near neutron stars (Barat 1984, Mazets 1980, Mitrofanov 1984, Nolan 1984).

A method of searching for spectral features in burst spectra (BATSE HER data) will be outlined in this paper. The method was used to investigate the energy range between approximately $350 \mathrm{keV}$ to $600 \mathrm{keV}$. This energy range was chosen because previous experiments have reported emission features in gamma-ray bursts around $400 \mathrm{keV}$ to $500 \mathrm{keV}$. These features have been interpreted as gravitationally red-shifted $511 \mathrm{keV}$ annihilation radiation produced near a neutron star (Barat 1984, Mazets 1980, Mitrofanov 1984, Nolan 1984).

The first step was to calculate a background model representing the ambient background radiation. The model was used to separate the burst spectrum from that of the background. Next, we construct the incident "photon" spectrum from the recorded "count" spectrum. To do this involves convolution with matrices that contain information on the detector's efficiency as a function of energy, as a function of angle of incidence of radiation, and also the detector's sensitivity to that fraction of the incident radiation caused by scattering off the Earth's atmosphere. The combination of all of these is called the detector response matrix (DRM) shown in Figure 1.

The BATSE HER data for a single burst can be binned into different time intervals and each interval forms a spectrum. Burst 1B 911221 was binned into 8 spectra each lasting approximately 9 secs. A fit of the spectrum that ranged in time from $9.7 \mathrm{secs}$ 
to 18.2 secs produced the best fit results. Figure 2 shows the fit that was made to this spectrum using a Broken Power Law, the form of which can be seen in Equation 1.

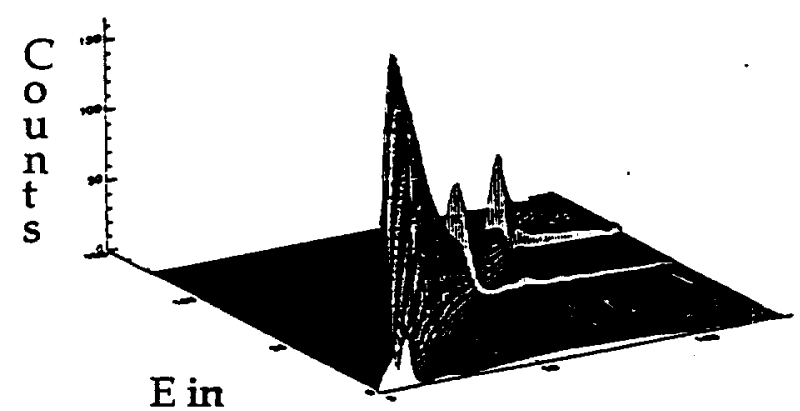

E out

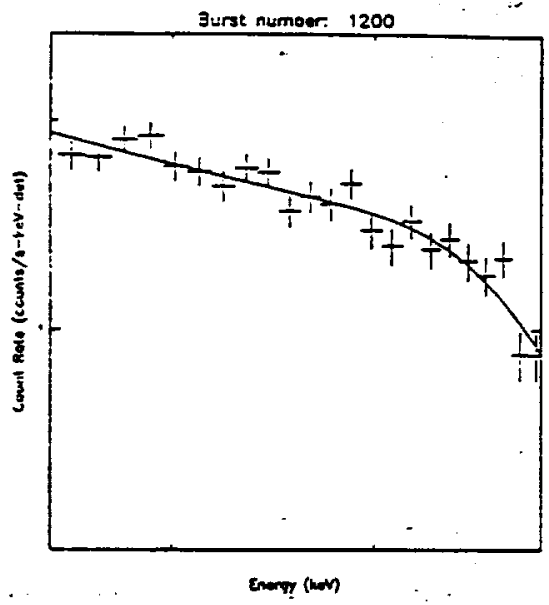

Figure 1 A detector response matrix. Figure 2 A fit using a Broken Power Law.

$$
\begin{aligned}
& A\left(\frac{E}{\text { Epivot }}\right)^{\lambda_{1}} \text { if } \mathrm{E} \leq \text { Ebreak } \\
& \text { or } \\
& A\left(\frac{\text { Ebreak }}{\text { Epivot }}\right)^{\lambda_{1}{ }^{-}}\left(\frac{E}{\text { Ebreak }}\right)^{\lambda_{2}} \text { if } \mathrm{E} \geq \text { Ebreak }
\end{aligned}
$$

The fit shown in Figure 2 produced a $\chi^{2}$ of 23.4 with 22 degrees of freedom. After the initial fit was made to this spectrum, a batch fit was made to the other 7 spectra by adjusting the parameters of the first fit to find the best fit for each of the others.

The batch fits form the basis of a continuum model which was then subtracted from the data. These residuals were then divided by the standard deviation, $\sigma$, that was associated with each energy value. Contour maps of the residuals plotted against energy and time were then generated. Figure 3 shows the contour map that was generated for burst 1B 911221.

The contour lines are displayed for values of $2 \sigma, 3 \sigma, 4 \sigma$, and $5 \sigma$. When examining the structure in contour plots the resolution of the detector at the particular energy must be considered in order to determine whether the structure is real or not. Equation 2 gives the resolution of the detector as a function energy. 


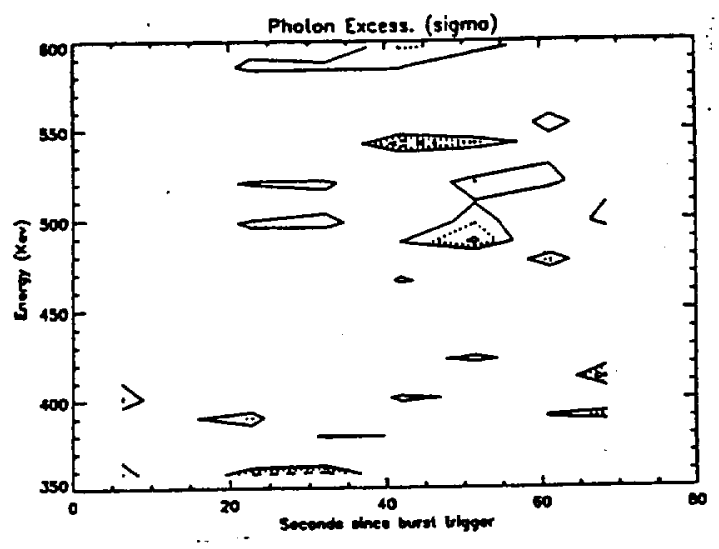

Figure 3 Contour map generated for burst 1B 911221.

$$
\operatorname{Res}=0.079 E\left(\frac{E}{511}\right)^{-0.42}
$$

At $545 \mathrm{keV}$ the resolution is $42 \mathrm{keV}$. Therefore, the structure seen at $545 \mathrm{keV}$ between 36 secs and 57 secs is probably a detector anomaly. The detector resolution at $490 \mathrm{keV}$ is $39 \mathrm{keV}$. The observed structure ranges from $480 \mathrm{keV}$ to $510 \mathrm{keV}$, so the feature is probably not real but further investigation is warranted. Figure 4 shows a plot over a larger energy range chosen to show the features at $490 \mathrm{keV}$ in the context of a larger continuum. The figure shows that, in the energy range of $480 \mathrm{keV}$ to 510 $\mathrm{keV}$, there are no significant features.

The feature searching method described above provides a means of searching through a vast amount of data, looking for regions which warrant further and more thorough searches.

The new searching method also allows us to evaluate our background subtracting and fitting routines. For instance, if there were a lot of structure around $511 \mathrm{keV}$ it might indicate that the background subtraction routines were not working properly.

Future work will be done to improve and enhance this searching method while analyzing GRB's for spectral emission features. 


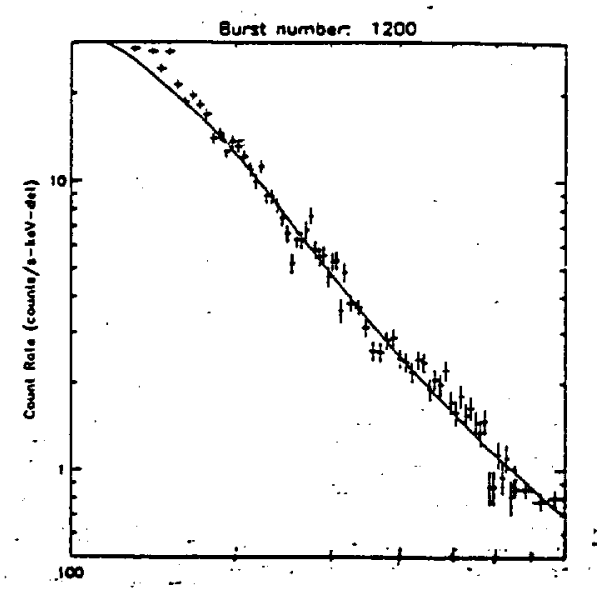

Figure 4 Fit of a broken power law over the energy range $170 \mathrm{keV}$ to $900 \mathrm{keV}$.

Acknowledgment:

I would like to thank the members of the BATSE group for unselfishly aiding me with my research especially G. J. Fishman, C. A. Meegan, M. S. Briggs, G. N. Pendleton, W. S. Paciesas, R. D. Preece, and M. N. Brock.

1. Barat, C.et al, Possible Short Annihilation Flashes in the 1978 November 4 Gamma-ray burst, The Astrophysical Journal, 286:L11-L13, November 1, 1984.

2. Hardin, A. K., Gamma-ray burst theory: back to the drawing board, ApJ, Supplement, January 11-15, 1993.

3. Mazets, E. P. et al, Lines in the Energy Spectra of Gamma-ray Bursts, Pis'ma Astron. Zh. 6,706-711, November 1980.

4. Mitrofanov, I. G. et al, Rapid Spectral Variability of Cosmic Gamma-ray Bursts, Astron. Zh. 61, 939-943, September-October, 1984.

5. Nolan, P. L. et al, Spectral Feature of 31 December $1981 \gamma$-ray Burst not Confirmed, Nature 311, September 27, 1984. 